\section{Niveles de colesterol no HDL en la población chilena y su asociación con enfermedad cardiovascular y diabetes mellitus: estudio de corte transversal en Encuesta Nacional de Salud 2009-2010}

\author{
MARÍA CONSUELO ALÉ ${ }^{1, a}$, GUADALUPE ECHEVERRÍA ${ }^{1,2, b}$, \\ ARANZAZU JUGO ${ }^{2, c}$, LUIS VILLARROEL ${ }^{3, d}$, \\ ALBERTO MAIZ ${ }^{2}$, ATTILIO RIGOTTI ${ }^{1,2, \mathrm{e}}$
}

\section{Non-HDL cholesterol levels in Chilean population and their association with diabetes mellitus and cardiovascular disease}

Background: Despite aggressive treatment aimed at lowering LDL cholesterol (LDL-C) levels with statins, there is a high residual prevalence of cardiovascular diseases, which may depend on plasma cholesterol transported in other atherogenic lipoproteins. Aims: To describe non-HDL cholesterol (non-HDL-C) levels in the Chilean population and their association with diabetes mellitus and cardiovascular disease. To evaluate compliance with non-HDL-C therapeutic goals-according to individual cardiovascular risk-at different levels of triglycerides, in comparison with $L D L-C$ goal achievement. Material and Methods: We analyzed data from 2,792 Chilean subjects aged $\geq 15$ years who were included in the 2009-2010 National Health Survey and had valid data for blood lipids, diabetes, and cardiovascular disease. Results: Forty five percent of subjects had high non-HDL-C levels. The proportion of diabetic and non-diabetic subjects with high non-HDL-C levels was 81 and $42 \%$, respectively $(p<0.01)$. A significant discordance was observed in the achievement of therapeutic objectives when $L D L-C$ or non-HDL-C levels were considered, particularly in presence of triglycerides $\geq 150 \mathrm{mg} / \mathrm{dl}$. Namely, $8 \%$ of the population showed elevated levels of high non-HDL-C despite adequate LDL-C levels. Conclusions: Evaluation and management of elevated non- $H D L-C$ in patients with adequate levels of $L D L-C$ seems worthwhile considering the discordance observed between these blood cholesterol fractions. This strategy may be effective to reduce the residual cardiovascular risk in the Chilean population.

(Rev Med Chile 2019; 147: 1365-1373)

Key words: Cardiovascular Diseases; Cholesterol; Cholesterol, VLDL; Diabetes Mellitus Type 2.
${ }^{1}$ Centro de Nutrición Molecular y Enfermedades Crónicas,

Escuela de Medicina, Pontificia Universidad Católica de Chile.

Santiago, Chile.

${ }^{2}$ Departamento de Nutrición,

Diabetes y Metabolismo,

Escuela de Medicina, Pontificia

Universidad Católica de Chile.

Santiago, Chile.

${ }^{3}$ Departamento de Salud Pública,

Escuela de Medicina, Pontificia

Universidad Católica de Chile.

Santiago, Chile.

${ }^{\mathrm{a} M D}$, Magíster en Nutrición

bIngeniero Civil Industrial,

Magíster en Ciencias de la

Ingeniería.

'MD, Magíster en Nutrición.

dEstadístico, PhD.

e MD, PhD.

Trabajo no recibió financiamiento. Los autores declaran no tener conflictos de interés.

Recibido el 31 de enero de 2019, aceptado el 30 de octubre de 2019.

Correspondencia a:

Dr. Attilio Rigotti

Departamento de Nutrición, Diabetes y Metabolismo,

Escuela de Medicina, Pontificia Universidad Católica de Chile Diagonal Paraguay 362, piso 4 . Santiago, CP 8330077, Chile. arigotti@med.puc.cl 


\begin{tabular}{|ll|}
\hline \multicolumn{2}{|l|}{ ABREVIATURAS } \\
\hline HDL $\quad:$ Lipoproteínas de alta densidad \\
\hline LDL $\quad:$ Lipoproteínas de baja densidad \\
\hline C-no HDL $:$ Colesterol no HDL \\
\hline C-LDL $\quad:$ Colesterol LDL \\
\hline C-VLDL & : Colesterol VLDL \\
\hline C-HDL & $:$ Colesterol HDL \\
\hline TG & $:$ Triglicéridos \\
\hline CT & $:$ Colesterol Total \\
\hline ECV $\quad:$ Enfermedad Cardiovascular \\
\hline RCV $\quad:$ Riesgo Cardiovascular \\
\hline DM2 & : Diabetes Mellitus tipo 2 \\
\hline ENS & $:$ Encuesta Nacional de Salud \\
\hline
\end{tabular}

L a consideración de los niveles de colesterol LDL (C-LDL) como objetivo terapéutico primario ha sido clave en la prevención de las enfermedades cardiovasculares $(\mathrm{ECV})^{1}$. La estimación de las cifras de C-LDL mediante la fórmula de Friedewald requiere la medición del colesterol total (CT), colesterol HDL (C-HDL) y los triglicéridos (TG) en ayuno ${ }^{2}$. Esta estimación del C-LDL es menos precisa en pacientes con TG altos, como ocurre en los pacientes diabéticos. De hecho, la fórmula no se debe aplicar con niveles de TG sanguíneos $>400 \mathrm{mg} / \mathrm{dl}^{2}$.

Por otro lado, y pese al tratamiento agresivo con estatinas y un mejor control del C-LDL, más de la mitad de los pacientes en tratamiento, vuelven a sufrir un nuevo evento cardiovascular ${ }^{3}$. Estos hallazgos indican la necesidad de complementar nuestros objetivos terapéuticos con el manejo de otros factores de riesgo más allá de los niveles de C-LDL.

El C-no HDL -que resulta de la simple diferencia aritmética entre el CT menos C-HDLestima todo el colesterol sanguíneo presente en lipoproteínas aterogénicas: VLDL, remanentes de VLDL y quilomicrones, lipoproteínas de densidad intermedia, LDL y Lp(a). Su cálculo es simple, no requiere muestras sanguíneas en ayuno y puede hacerse con cualquier nivel de TG sanguíneos ${ }^{4}$.

Los niveles de C-no HDL son un buen predictor de riesgo cardiovascular $(\mathrm{RCV})^{6}$. Por ejemplo, el C-no HDL es un mejor predictor independiente de infarto agudo al miocardio no fatal ${ }^{7} \mathrm{y}$ de riesgo de enfermedad coronaria futura ${ }^{8}$ comparado con los niveles de C-LDL. Incluso en pacientes tratados adecuadamente con estatinas, el C-no HDL predice de mejor forma el riesgo de re-incidencia de eventos cardiovasculares en comparación con el C-LDL 9 .

La medición y utilización de esta fracción de colesterol sanguíneo podría ser útil en el manejo del riesgo cardiovascular residual ya que las ECV siguen siendo la primera causa de muerte en Chile y el mundo ${ }^{10}$. Además, nuestro país mostró un incremento del síndrome metabólico y diabetes mellitus tipo 2 (DM2) desde 2003 a 2009-2010 ${ }^{11,12}$. Estas condiciones presentan insulinorresistencia con trastornos lipídicos caracterizados por una dislipidemia aterogénica que se detectaría mejor evaluando el C-no HDL en comparación con la estimación del C-LDL ${ }^{5}$.

El objetivo de este estudio fue describir los niveles de C-no HDL según distintas variables sociodemográficas y clínicas y evidenciar la prevalencia de C-no HDL elevado y su asociación con ECV y la DM2 en la población chilena evaluada en la Encuesta Nacional de Salud (ENS) 2009-2010.

\section{Material y Métodos}

\section{Muestra estudiada}

La ENS 2009-2010 fue un estudio de corte transversal que realizó un muestreo probabilístico multietápico y estratificado con representatividad nacional y por zona rural y urbana. La población objetivo fueron individuos $\geq 15$ años de edad no institucionalizados.

Para el presente estudio, se analizó una submuestra aleatoria de 2.792 sujetos $\geq 15$ años con datos válidos para C-HDL, CT, triglicéridos, diabetes y ECV. En cuanto a pacientes diabéticos, se incluyeron participantes con glicemia $\geq 126$ $\mathrm{mg} / \mathrm{dl}$ en ayuno $\geq 8 \mathrm{~h}$ y/o con diagnóstico previo de diabetes. Para ECV, se aplicó un cuestionario abreviado modificado de angina y además se incluyeron a los pacientes con diagnóstico y/o tratamiento previos de ECV.

El protocolo y el consentimiento informado de esta encuesta fueron aprobados por el Comité de Ética en Investigación de la Pontificia Universidad Católica de Chile y del Ministerio de Salud y el estudio fue ejecutado por el Departamento de Salud Pública de la misma universidad. 


\section{Recolección de datos y análisis de laboratorio}

La encuesta incluyó dos visitas domiciliarias en que se aplicaron cuestionarios de salud (antecedentes mórbidos personales, familiares y tratamientos farmacológicos), se realizó un examen físico y se tomaron muestras de sangre en ayunas. Además se recolectaron hábitos de estilo de vida (tabaquismo, actividad física y alimentación) mediante cuestionarios predefinidos por la ENS 2009-2010 ${ }^{12}$. Las muestras fueron procesadas y analizadas en el Laboratorio Central del Hospital Clínico de la Pontificia Universidad Católica de Chile. CT, C-HDL y glicemia fueron medidos con métodos estandarizados. Los niveles de C-LDL se estimaron según la fórmula de Friedewald ${ }^{2}$. Los niveles de C-no HDL se calcularon a partir de la diferencia entre el CT y el C-HDL.

\section{Estimación del RCV individual}

La estimación del RCV individual se realizó según recomendaciones de la guía MINSAL $2014^{13}$. Por medio de la anamnesis y exámenes de laboratorio, se identificó un primer grupo de alto riesgo correspondiente a pacientes con factores de riesgo y patologías que determinan una alta probabilidad de sufrir un evento cardiovascular. Al resto de los individuos, se calculó su RCV a 10 años por medio de fórmulas calibradas y validadas para nuestra población ${ }^{14}$. A los pacientes clasificados en categorías de riesgo moderado y bajo, se evaluó si presentaban síndrome metabólico o antecedentes familiares de ECV prematura para aumentar al siguiente nivel de RCV. De esta forma, la población quedó categorizada en RCV bajo, moderado y alto.

\section{Definición de metas del C-no HDL}

Las metas terapéuticas para este parámetro lipídico se definieron como cifras $30 \mathrm{mg} / \mathrm{dl}$ superiores a los valores establecidos como objetivos terapéuticos del C-LDL para cada categoría de RCV por la guía MINSAL $2014^{13}$ según la recomendación de guías internacionales ${ }^{1}$.

\section{Análisis estadístico}

Todos los análisis estadísticos se hicieron considerando un estudio de muestras complejas. Los datos de la muestra original fueron corregidos por factores de expansión para que la muestra expandida fuera equivalente a la población chilena $\geq 15$ años de edad proyectada según el censo al año 2010.
Las variables continuas o categóricas se compararon con las pruebas t de Student o Chi cuadrado, respectivamente. Las correlaciones entre los niveles de C-no HDL y LDL se evaluaron por el coeficiente de correlación de Pearson. Las asociaciones entre C-no HDL y prevalencia de DM2 y ECV se analizaron en modelos de regresión logística compleja, ajustados por sexo, edad, zona de residencia, nivel educacional y otros factores como IMC, hábito tabáquico, nivel de actividad física, calidad de la dieta y síndrome metabólico. Una diferencia, asociación o correlación se consideró estadísticamente significativa cuando el valor $\mathrm{p}$ resultó $<0,05$.

Los análisis fueron realizados en el programa estadístico SPSS v15.0 para Windows.

\section{Resultados}

\section{Características de la muestra de estudio}

La muestra de estudio consistió en 2.792 personas $\geq 15$ años de edad siendo representativa de la población adulta chilena, con más mujeres $(51,1 \%)$ que hombres $(48,9 \%)$ y una edad promedio de la muestra de 41,7 años. Las prevalencias de DM2 y ECV fueron de $8 \%$ y $10,9 \%$, respectivamente. La Tabla 1 muestra las características clínicas y sociodemográficas de la muestra estudiada.

\section{Correlación entre los niveles de C-no HDL con $C-L D L$ según presencia de TG normales vs elevados}

La Figura 1 muestra la relación entre los niveles de C-no HDL y C-LDL según la coexistencia TG $<150 \mathrm{mg} / \mathrm{dl} \mathrm{o} \geq 150 \mathrm{mg} / \mathrm{dl}$. La correlación entre estas variables en presencia de TG normales mostró una asociación positiva lineal con $\mathrm{R}^{2}=0,975$. Cuando analizamos esta misma correlación en contexto de $\mathrm{TG} \geq 150 \mathrm{mg} / \mathrm{dl}$, la asociación se mantuvo positiva, pero fue menos estrecha $y$ disminuyó a $\mathrm{R}^{2}=0,696$.

\section{Niveles de C-no HDL en la población chilena según variables sociodemográficas y clínicas}

En primer lugar, el promedio global del C-no HDL en la población analizada fue $141,8 \mathrm{mg} / \mathrm{dl}$ (IC: 139,0-144,6) con una distribución no normal $(\mathrm{p}=0,041)$.

En cuanto a la relación con variables sociodemográficas (Tabla 2), los hombres presentaron 
Tabla 1. Descripción de la muestra expandida de la ENS Chile 2009-2010 ( $n=2.792)$ utilizada en el análisis de niveles del colesterol no HDL

\begin{tabular}{|c|c|c|}
\hline & $\begin{array}{l}\text { Recuento } \\
\text { no ponde- } \\
\text { rado }\end{array}$ & $\begin{array}{c}\text { Frecuencia } \\
\text { expandida } \\
(\%)\end{array}$ \\
\hline \multicolumn{3}{|l|}{ Según sexo } \\
\hline Hombres & 1.150 & $48,9 \%$ \\
\hline Mujeres & 1.642 & $51,1 \%$ \\
\hline \multicolumn{3}{|l|}{ Según grupos de edad } \\
\hline 15 a 24 años & 411 & $21,5 \%$ \\
\hline 25 a 44 años & 924 & $38,7 \%$ \\
\hline 45 a 64 años & 908 & $27,4 \%$ \\
\hline 65 y más años & 549 & $12,5 \%$ \\
\hline \multicolumn{3}{|l|}{ Según nivel educacional } \\
\hline Bajo & 702 & $17,5 \%$ \\
\hline Medio & 1.503 & $55,4 \%$ \\
\hline Alto & 525 & $27,2 \%$ \\
\hline \multicolumn{3}{|l|}{ Según zona de residencia } \\
\hline Urbana & 2.422 & $90,0 \%$ \\
\hline Rural & 370 & $10,0 \%$ \\
\hline \multicolumn{3}{|l|}{ Según estado nutricional } \\
\hline Bajo peso & 48 & $1,6 \%$ \\
\hline Peso normal & 812 & $35,8 \%$ \\
\hline Sobrepeso & 1.048 & $36,3 \%$ \\
\hline Obesidad & 808 & $26,3 \%$ \\
\hline \multicolumn{3}{|l|}{ Según hábito tabáquico } \\
\hline No fumador & 1.790 & $60,3 \%$ \\
\hline Fumador actual & 939 & $39,7 \%$ \\
\hline \multicolumn{3}{|l|}{ Según nivel de actividad física } \\
\hline Bajo & 941 & $28,4 \%$ \\
\hline Medio & 516 & $18,6 \%$ \\
\hline Alto & 1.273 & $53,0 \%$ \\
\hline \multicolumn{3}{|l|}{ Según calidad de la dieta } \\
\hline Saludable & 135 & $5,5 \%$ \\
\hline No saludable & 2.591 & $94,5 \%$ \\
\hline \multicolumn{3}{|l|}{ Dislipidemias } \\
\hline Colesterol total > $200 \mathrm{mg} / \mathrm{dl}$ & 1.209 & $38,5 \%$ \\
\hline Colesterol LDL > 130 mg/dl & 947 & $29,4 \%$ \\
\hline Colesterol HDL $<40 / 50$ mg/dl & 1.323 & $45,4 \%$ \\
\hline Triglicéridos > 150 mg/dl & 1.108 & $35,3 \%$ \\
\hline \multicolumn{3}{|l|}{ Sospecha de Diabetes } \\
\hline No & 2.457 & $92,0 \%$ \\
\hline Sí & 252 & $8,0 \%$ \\
\hline \multicolumn{3}{|l|}{ Enfermedad cardiovascular } \\
\hline No & 2.382 & $89,1 \%$ \\
\hline Sí & 341 & $10,9 \%$ \\
\hline
\end{tabular}

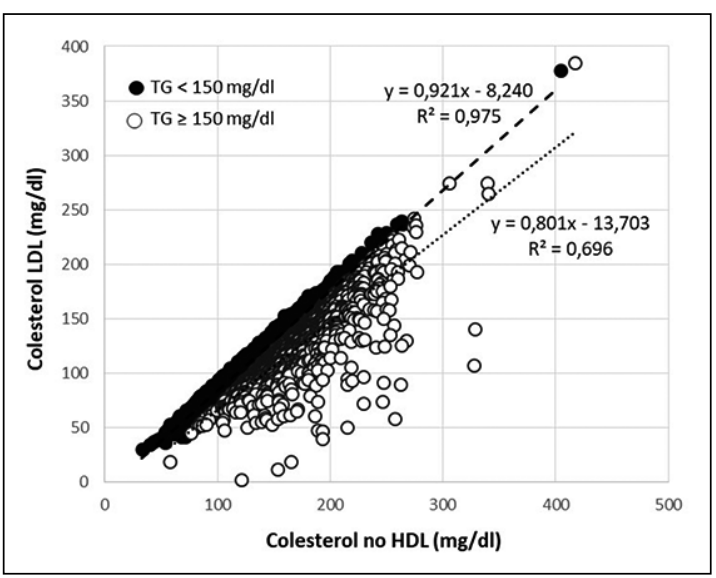

Figura 1. Correlación entre los niveles de C-no HDL y C-LDL según la presencia de TG normales o elevados en la población chilena.

una media de C-no HDL (144,9 mg/dl) mayor que las mujeres $(138,9 \mathrm{mg} / \mathrm{dl} ; \mathrm{p}=0,036)$. Según grupos de edad, los niveles de C-no HDL presentaron diferencias significativas ( $\mathrm{p}<0,001)$, siendo el grupo etario de 45 a 64 años el que evidenció mayor nivel de C-no HDL (163 mg/dl).

En cuanto a variables clínicas (Tabla 2), los pacientes con obesidad exhibieron mayores niveles de C-no HDL ( $156 \mathrm{mg} / \mathrm{dl}$ ) versus los pacientes de peso normal $(128,7 \mathrm{mg} / \mathrm{dl}$; $\mathrm{p}<0,001)$. Por otro lado, los pacientes con diabetes y ECV presentaron niveles significativamente mayores $(\mathrm{p}<0,001 \mathrm{y}$ $\mathrm{p}=0,002$ respectivamente) de C-no HDL que los pacientes que no presentaban estas enfermedades.

Prevalencia de C-no HDL elevado según distintas variables sociodemográficas y clínicas

A nivel global, el porcentaje de C-no HDL elevado en la población estudiada según el RCV estimado para cada individuo fue $45,1 \%$. La Figura 2 muestra los niveles del C-no HDL elevado según diferentes variables sociodemográficas. En cuanto a la edad, el grupo de 45 a 64 años presentó el mayor porcentaje de C-no HDL alto $(69,1 \%$, $\mathrm{p}<0,001)$. En cuanto a nivel educacional, los pacientes con menor nivel educacional exhibieron mayor prevalencia de C-no HDL elevado (57,4\%, $\mathrm{p}=0,004)$.

En relación a variables clínicas (Figura 3), los pacientes obesos presentaron la mayor frecuencia de C-no HDL alto $(47,8 \%, \mathrm{p}<0,001)$ en comparación al resto de las categorías de estado nutricional. 
Tabla 2. Niveles de colesterol no HDL y colesterol LDL según variables sociodemográficas y clínicas en Chile

\begin{tabular}{|c|c|c|c|c|}
\hline \multirow[b]{2}{*}{ Time } & \multicolumn{2}{|c|}{ Colesterol no HDL } & \multicolumn{2}{|c|}{ Colesterol LDL } \\
\hline & Media (IC 95\%) & valor $\mathbf{p}$ & Media (IC 95\%) & valor $\mathbf{p}$ \\
\hline \multicolumn{5}{|l|}{ Según sexo: } \\
\hline Hombres & $144,9(140,3-149,5)$ & 0,036 & $113,5(109,7-117,2)$ & 0,973 \\
\hline Mujeres & $138,9(135,6-142,1)$ & & $113,4(110,9-115,9)$ & \\
\hline \multicolumn{5}{|c|}{ Según grupos de edad } \\
\hline 15 a 24 años & $112,4(108,3-116,5)$ & $<0,001$ & $91,4(88,3-94,5)$ & $<0,001$ \\
\hline 25 a 44 años & $142,4(138,0-146,9)$ & & $113,6(110,2-117,1)$ & \\
\hline 45 a 64 años & $163,0(157,7-168,3)$ & & $128,8(124,2-133,3)$ & \\
\hline 65 y más años & $144,2(138,5-150,0)$ & & $117,0(112,2-121,8)$ & \\
\hline \multicolumn{5}{|c|}{ Según nivel educacional } \\
\hline Bajo & $147,0(141,8-152,3)$ & 0,075 & $117,5(113,3-121,6)$ & 0,096 \\
\hline Medio & $141,9(137,8-145,9)$ & & $113,3(110,1-116,6)$ & \\
\hline Alto & $138,5(133,2-143,8)$ & & $111,2(107,1-115,2)$ & \\
\hline \multicolumn{5}{|c|}{ Según zona de residencia } \\
\hline Urbana & $141,6(138,5-144,7)$ & 0,518 & $113,1(110,6-115,5)$ & 0,185 \\
\hline Rural & $143,7(138,1-149,3)$ & & $116,8(111,8-121,8)$ & \\
\hline \multicolumn{5}{|c|}{ Según estado nutricional } \\
\hline Bajo peso & $105,8(91,1-120,4)$ & $<0,001$ & $89,8(78,0-101,7)$ & $<0,001$ \\
\hline Peso normal & $128,7(123,3-134,0)$ & & $106,6(102,3-110,8)$ & \\
\hline Sobrepeso & $146,7(142,3-151,2)$ & & $117,3(113,6-121,0)$ & \\
\hline Obesidad & $156,0(151,0-160,9)$ & & $119,6(115,9-123,4)$ & \\
\hline \multicolumn{5}{|c|}{ Sospecha de Diabetes } \\
\hline No & $140,2(137,3-143,1)$ & $<0,001$ & $112,9(110,6-115,3)$ & 0,139 \\
\hline Sí & $158,8(149,0-168,5)$ & & $119,0(111,3-126,7)$ & \\
\hline \multicolumn{5}{|c|}{ Enfermedad cardiovascular } \\
\hline No & $140,3(137,3-143,4)$ & 0,002 & $112,9(110,5-115,3)$ & 0,205 \\
\hline Sí & $153,9(145,8-162,0)$ & & $117,3(111,0-123,7)$ & \\
\hline
\end{tabular}

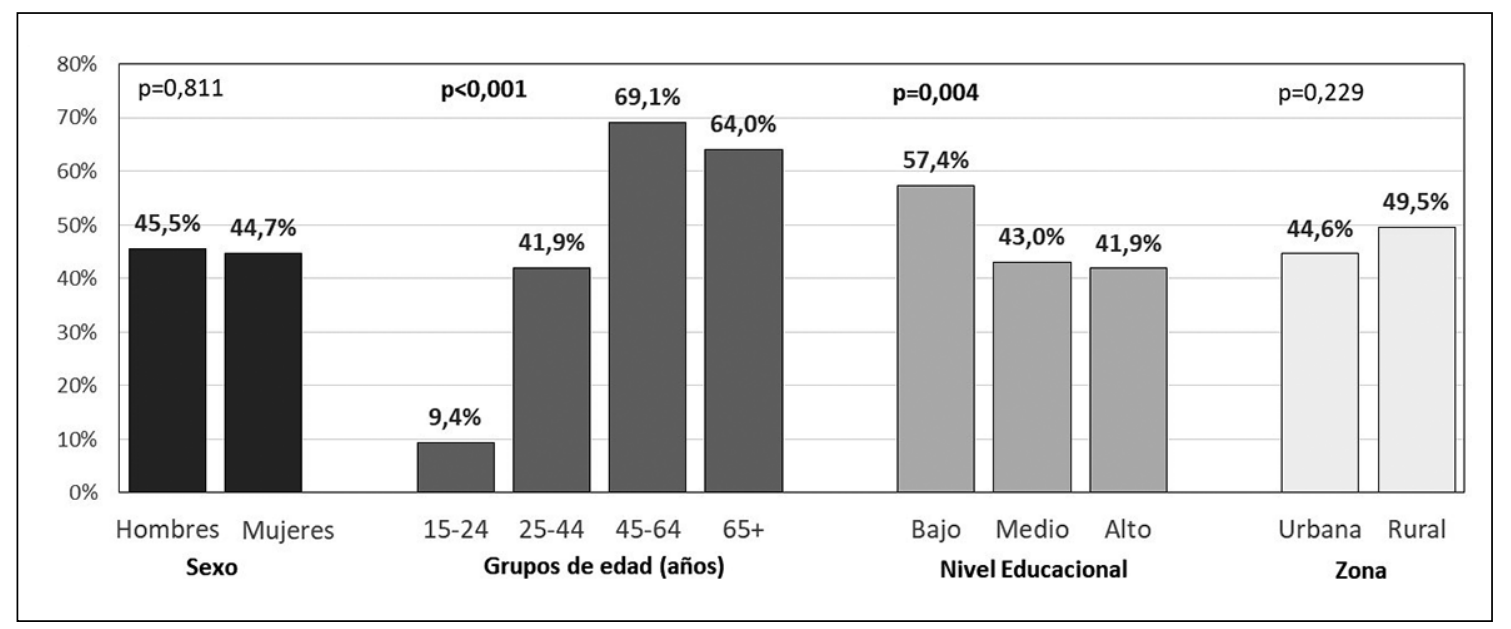

Figura 2. Frecuencia de C-no HDL elevado en la población chilena según diferentes variables sociodemográficas. 


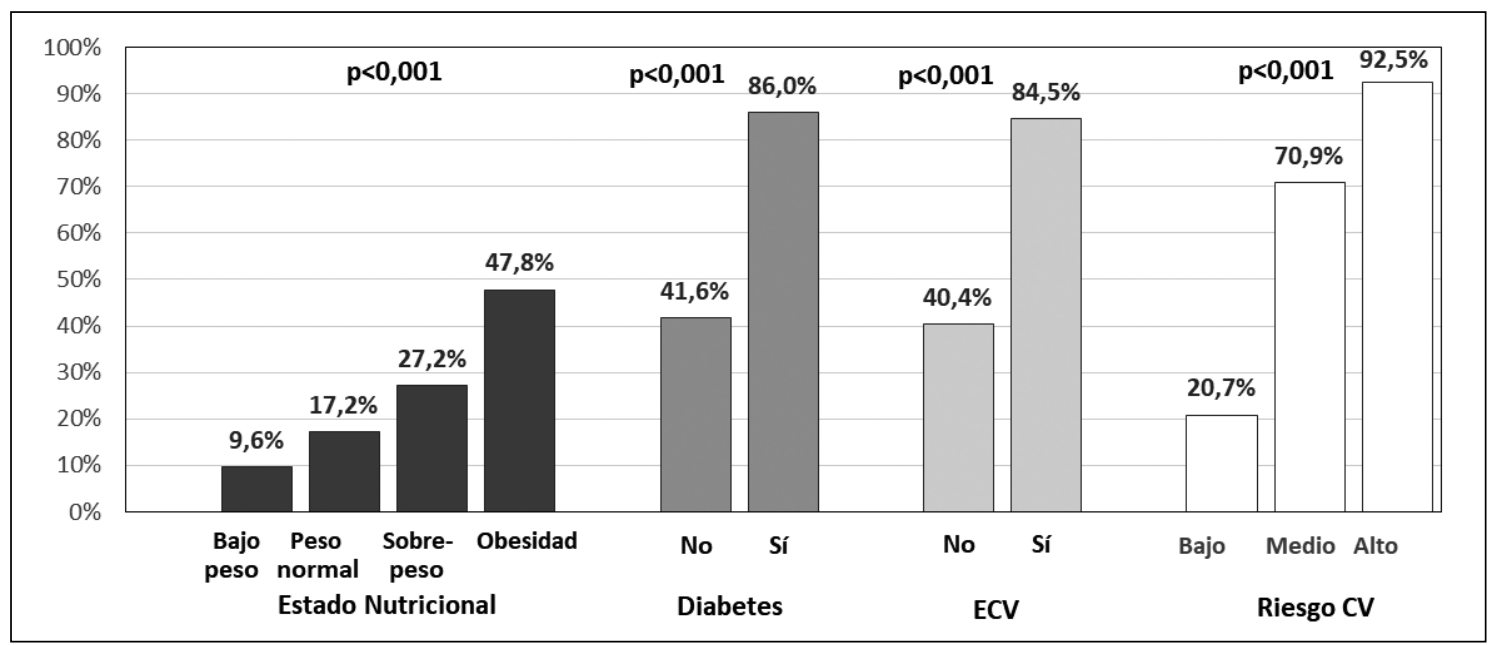

Figura 3. Frecuencia de C-no HDL elevado en la población chilena según diferentes variables clínicas.

Un $86 \%$ de los pacientes con diabetes presentaron niveles elevados de esta fracción de colesterol versus $41,6 \%$ para sujetos no diabéticos. En los pacientes con ECV, 84,5\% de ellos presentaron C-no HDL elevado versus $40,4 \%$ en sujetos sin este antecedente.

En cuanto al cumplimiento de las metas de C-no HDL para los distintos niveles de RCV definidos por la guía MINSAL 2014, los pacientes que presentaban RCV bajo tuvieron un cumplimiento aceptable $(79,3 \%)$ de la meta $<160 \mathrm{mg} / \mathrm{dl}$. En el caso de los pacientes con RCV moderado, 29,1\% de los pacientes cumplían la meta de C-no HDL $<130 \mathrm{mg} / \mathrm{dl}$ para este riesgo. Por último, los individuos con alto RCV mostraron el menor cumplimiento -solamente 7,5\%- de la meta recomendada $(<100 \mathrm{mg} / \mathrm{dl})$ para este parámetro lipídico.

\section{Asociación de diabetes mellitus o ECV con la presencia de niveles elevados de C-LDL y C-no $H D L$}

A continuación, se analizó la presencia DM2 o ECV según la existencia de niveles elevados o normales de C-LDL y C-no HDL aplicando 3 modelos estadísticos con ajuste progresivo para controlar por posibles variables confundentes (Figura 4).

Los resultados mostraron una asociación positiva tanto para DM2 (modelo 1: $\mathrm{OR}=4,59, \mathrm{IC} 95 \%$ 2,17-9,69, $\mathrm{p}<0,001$; modelo 2: OR $=3,67$, IC95\% $1,71-7,88 ; \mathrm{p}=0,001 ;$ modelo $3: \mathrm{OR}=2,69, \mathrm{IC} 95 \%$ $1,17-6,17, \mathrm{p}<0,001$ ) como para ECV (modelo 1:
$\mathrm{OR}=6,22$, IC95\% 3,56-10,86, $\mathrm{p}<0,001$; modelo 2: $\mathrm{OR}=7,08$, IC95\% 3,81-13,14, $\mathrm{p}<0,001$, modelo 3: $\mathrm{OR}=5,89, \mathrm{IC} 95 \% 3,11-11,15, \mathrm{p}=0,008) \mathrm{con}$ la presencia de niveles altos de C-LDL (Figura 4).

$\mathrm{Al}$ analizar la razón de chance para la presencia diabetes o ECV según los niveles de C-no HDL (Figura 4) elevado versus no elevado, también se evidenció una asociación significativa tanto para DM2 (modelo 1: OR = 5,54, IC95\% 2,73-11,29; $\mathrm{p}<0,001$, modelo 2: OR $=4,32$, IC95\% 2,07$9,10, \mathrm{p}<0,001$ y modelo 3: OR = 2,99, IC95\% 1,30-6,92, $\mathrm{p}<0,001$ ) como para ECV (modelo 1 : $\mathrm{OR}=6,12$, IC95\% 3,64-10,30; $<<0,001$; modelo $2:$ OR $=6,44$, IC95\% 3,71-11,18, p < 0,001 y modelo 3: OR = 5,57, IC95\% 3,05-10,176, p = 0,04).

\section{Discusión}

Este estudio tuvo como principal objetivo describir los niveles de C-no HDL en Chile usando una muestra representativa obtenida de la ENS 2009-2010, dado que no existen otros análisis similares sobre este parámetro lipídico en nuestro país.

Como ha sido reportado y también obtuvimos de nuestros propios resultados, la correlación entre los niveles de C-no HDL y C-LDL disminuye cuando existe hipertrigliceridemia, generando una discrepancia significativa entre el logro de objetivos terapéuticos cuando se consideran únicamente los valores de C-LDL versus las cifras de C-no HDL. Extrapolando a la proyección de la población 


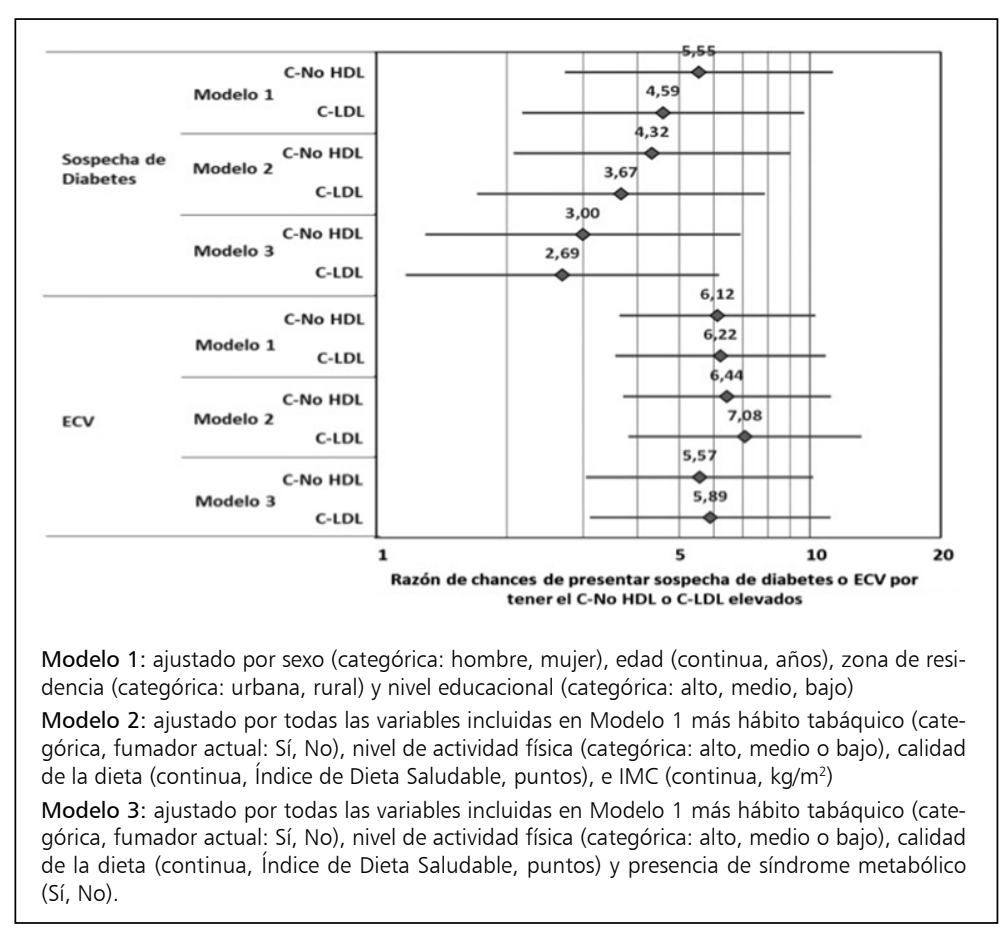

Figura 4. Razón de chance para la asociación de diabetes mellitus o enfermedad cardiovascular con la presencia de niveles de C-no HDL y C-LDL elevados versus normales en la población chilena. chilena correspondiente al censo del año 2010, 2,7\% (203.765 participantes) presentaron niveles de C-LDL dentro de límites normales, aunque al mismo tiempo exhibían cifras de C-no HDL elevado, mientras que 5,7\% (430.100 participantes) mostraron valores de C-LDL elevado, pero C-no HDL dentro de límites normales. Por otro lado, si separamos la población estudiada en sujetos con TG $<150$ versus $\geq 150 \mathrm{mg} / \mathrm{dl}$, ningún participante con TG y C-LDL normales presentó cifras de C-no HDL elevado. En el caso de TG $\geq 150 \mathrm{mg} / \mathrm{dl}, 8 \%$ $(n=203.763)$ de los sujetos que exhibieron C-LDL dentro de los límites recomendados presentaban niveles de C-no HDL incrementados. Esta misma discrepancia entre el logro de metas de C-LDL y C-no HDL se observó en EE.UU. ${ }^{16}$. Estos hallazgos evidencian una discordancia significativa en la prevalencia de dislipidemia por C-LDL y C-no HDL según los niveles de triglicéridos.

El análisis de dos estudios prospectivos, randomizados y multicéntricos (TNT (Treating to New Targets) e IDEAL (Incremental Decrease End Points Through Aggressive Lipid Lowering)) evidenciaron que los niveles de C-no HDL en pacientes en tratamiento con estatinas eran un mejor predictor de
RCV residual que las cifras estimadas de C-LDL 9 . El mismo hallazgo fue reportado como resultado de un metaanálisis con 38.153 pacientes en tratamiento con estatinas ${ }^{17}$, en el cual los niveles de C-no HDL mostraron una asociación más fuerte y de mejor valor predictivo de nuevos eventos cardiovasculares mayores en comparación con el C-LDL. Por otro lado, la razón CT/C-no HDL también se ha asociado más significativamente a aterosclerosis subclínica en comparación con otros parámetros lipídicos en Chile ${ }^{18}$.

En pacientes con hipertrigliceridemia (TG $\geq 150 \mathrm{mg} / \mathrm{dl}$ ), es recomendable considerar al C-no HDL como marcador de dislipidemia aterogénica ${ }^{1}$. En especial, pacientes con perfil cardiometabólico de resistencia insulínica, síndrome metabólico y/o DM2 serían los más beneficiados de considerar los valores de C-no HDL como otro objetivo terapéutico en su evaluación y seguimiento periódico ${ }^{7,19}$. De hecho, la American Diabetes Association (ADA) $\mathrm{y}$ el American College of Cardiology (ACC) establecen que la medición del C-no HDL es una mejor medida que el C-LDL para identificar pacientes con un alto riesgo, especialmente aquellos que presentan múltiples factores de riesgo metabólicos ${ }^{20}$. 
Por último, demostramos una asociación entre la existencia de C-no HDL o C-LDL elevados y la presencia de DM2 o ECV ajustando por distintas variables, incluyendo el síndrome metabólico (modelo 3 de ajuste). Incluso, se ha reportado previamente que pacientes con C-no HDL elevado y C-LDL normal tienen chance aumentada de presencia de diabetes y mayor $\mathrm{RCV}^{16}$. Los niveles elevados de C-no HDL indican la existencia de remanentes de lipoproteínas aterogénicas que favorecen la aparición de partículas de LDL pequeñas y densas en los pacientes con resistencia a la insulina, síndrome metabólico, obesidad y diabetes.

Este estudio tiene como ventajas el uso de una muestra de tamaño considerable y representativa de sujetos chilenos, tomas y análisis de las muestras sanguíneas en forma protocolizadas, disponibilidad de parámetros adicionales para ajustes de las asociaciones observadas, todo lo cual genera buen grado de confiabilidad y extrapolabilidad de los hallazgos hacia la población general de nuestro país. Sin embargo, los hallazgos de asociación transversal no permiten establecer relaciones de riesgo en comparación con estudios observacionales longitudinales ni de causalidad como ocurriría en un estudio de intervención. Esta limitación no permite establecer puntos de corte locales para el manejo clínico de los niveles de C-no HDL. Por otro lado, las cifras de C-LDL y C-no HDL disponibles derivan de una medición única y no considera la variabilidad intraindividual que puede observarse entre diferentes tomas de muestras.

En conclusión, este estudio corresponde al primer reporte de las cifras de C-no HDL -un factor de RCV emergente- en Chile y su asociación con variables sociodemográficas y clínicas. Adicionalmente, un importante número de chilenos que muestran niveles de C-LDL dentro de límites normales simultáneamente presentan niveles C-no HDL elevado -especialmente en el contexto de TG $>150 \mathrm{mg} / \mathrm{dl}$. Por lo tanto, un número significativo de nuestra población queda desprotegida y sin posibilidad de disminuir su RCV por medio del uso de estatinas centrado solamente en el control del C-LDL. Dada la alta prevalencia de ECV y considerando que son la primera causa de mortalidad en nuestro país ${ }^{10}$, el cálculo del C-no HDL parece ser una excelente alternativa como objetivo terapéutico adicional, y sus metas deben también perseguirse de manera agresiva junto al logro del objetivo terapéutico para el C-LDL.
Agradecimientos: Departamento de Salud Pública de la Pontifica Universidad Católica de Chile por facilitarnos la base de datos que se utilizó en este estudio. Ministerio de Salud de Chile por el financiamiento de ENS 2009-2010.

\section{Referencias}

1. National Cholesterol Education Program (NCEP) Expert Panel on Detection, Evaluation, and Treatment of High Blood Cholesterol in Adults (Adult Treatment Panel III). Third Report of the National. Circulation 2002; 106: 3143-421.

2. Friedewald WT, Levy RI, Fredrickson DS. Estimation of the concentration of low-density lipoprotein cholesterol in plasma, without use of the preparative ultracentrifuge. Clin Chem 1972; 18: 499-552.

3. Lloyd-Jones D, Adams RJ, Brown TM, Carnethon M, Dai S, De Simone G, et al. American Heart Association. Executive summary: heart disease and stroke statistics--2010 update: a report from the American Heart Association. Circulation 2010; 121: 948-54.

4. Frost PH, Havel RJ. Rationale for use of non-high-density lipoprotein cholesterol rather than low-density lipoprotein cholesterol as a tool for lipoprotein cholesterol screening and assessment of risk and therapy. Am J Cardiol 1998; 81: 26B-31B.

5. Grundy M. Scott, Becker D, Clark L. Third Report of the National Cholesterol Education Program (NCEP) Expert Panel on Detection, Evaluation, and Treatment of High Blood Cholesterol in Adults (Adult Treatment Panel III) final report. Circulation 2002; 106: 3143-421.

6. Cui Y, Blumenthal RS, Flaws JA, Whiteman MK, Langenberg P, Bachorik PS, et al. Non-high-density lipoprotein cholesterol level as a predictor of cardiovascular disease mortality. Arch Intern Med 2001; 161: 1413-9.

7. Bittner V, Hardison R, Kelsey SF. Non-high-density lipoprotein cholesterol levels predict five-year outcome in the Bypass Angioplasty Revascularization Investigation (BARI). Circulation 2002; 106: 2537-42.

8. Arsenault BJ, Rana JS, Stroes ES, Despres JP, Shah PK, Kastelein JJ, et al. Beyond low-density lipoprotein cholesterol: respective contributions of non-high-density lipoprotein cholesterol levels, triglycerides, and the total cholesterol/high-density lipoprotein cholesterol ratio to coronary heart disease risk in apparently healthy men and women. J Am Coll Cardiol 2009; 55: 35-41.

9. Kastelein JJ, Van der Steeg WA, Holme I, Gaffney M, Cater NB, Barter P, et al. TNT Study Group; IDEAL Study Group. Lipids, apolipoproteins, and their ratios in 
relation to cardiovascular events with statin treatment. Circulation 2008; 117: 3002-9.

10. DEIS-MINSAL. Indicadores Básicos de Salud Chile. 2014, http://www.deis.cl/indicadores-basicos-de-salud/

11. Encuesta Nacional de Salud Chile, 2003, http://www. medicinadefamiliares.cl/Protocolos/encnacsalres.pdf.

12. Encuesta Nacional de Salud Chile, 2009-2010, http:// web.minsal.cl/portal/url/item/bcb03d7bc28b64dfe040010165012d23.pdf.

13. Consenso para enfoque de riesgo para la prevención de enfermedades cardiovasculares. MINSAL, Chile, 2014.

14. Kunstmann S, Lira MR, et al. Validación de tablas de riesgo cardiovascular adaptadas a la población chilena y su comparación con instrumentos internacionales, 2008. http://pifrecv.utalca.cl/docs/2008/Informe_Final_Fonis_SA06I2006_Web.pdf.

15. Expert Panel (Adult Treatment Panel II). Detection, evaluation, and treatment of high blood cholesterol in adults. Circulation 1994; 89: 1329-445.

16. Kilgore M, Muntner P, Woolley M, Sharma P, Bittner
V, Rosenson RS. Discordance between high non-HDL cholesterol and high LDL-cholesterol among US adults. J Clin Lipidol 2014; 8: 86-93.

17. Boekholdt SM, Arsenault BJ, Mora S, Pedersen TR, LaRosa JC, Nestel PJ, et al. Association of LDL cholesterol, non-HDL cholesterol, and apolipoprotein B levels with risk of cardiovascular events among patients treated with statins: a meta-analysis. JAMA 2012; 307: 1302-9.

18. Acevedo M, Kramer V, Tagle R, Corbalán R, Arnaíz P, Berríos X, et al. Total/HDL cholesterol ratio and non HDL cholesterols predictors for increase intima media thickness. Rev Med Chile 2012; 140: 969-76.

19. Lu W, Resnick HE, Jablonski KA, Jones KL, Jain AK, Howard WJ, et al. Non-HDL cholesterol as a predictor of cardiovascular disease in type 2 diabetes: the strong heart study. Diabetes Care 2003; 26: 16-23.

20. Robinson JG, Wang S, Smith BJ, Jacobson TA. Meta-analysis of the relationship between non-high-density lipoprotein cholesterol reduction and coronary heart disease risk. J Am Coll Cardiol 2009; 53: 316-22. 